\title{
Shrinkage simplex-centroid designs for a quadratic mixture model
}

\author{
Taha Hasan $^{1} \cdot$ Sajid Ali $^{2} \cdot$ Munir Ahmed ${ }^{3}$
}

Received: 21 March 2015/Accepted: 5 June 2017/Published online: 19 June 2017

(c) The Author(s) 2017. This article is an open access publication

\begin{abstract}
A simplex-centroid design for $q$ mixture components comprises of all possible subsets of the $q$ components, present in equal proportions. The design does not contain full mixture blends except the overall centroid. In real-life situations, all mixture blends comprise of at least a minimum proportion of each component. Here, we introduce simplex-centroid designs which contain complete blends but with some loss in $D$-efficiency and stability in $G$-efficiency. We call such designs as shrinkage simplexcentroid designs. Furthermore, we use the proposed designs to generate component-amount designs by their projection.
\end{abstract}

Keywords Mixture experiment · Simplex-centroid design $\cdot$ D-optimality $\cdot$ G-optimality

\section{Introduction}

In a mixture experiment with $q$ components, the proportion of ingredients may be denoted by $x_{1}, x_{2}, \ldots, x_{q}$, where $x_{i} \geq 0$ for $i=1,2, \ldots, q$ and $\sum_{i=1}^{q} x_{i}=1$. The response

\section{Taha Hasan}

taha.qau@gmail.com

Sajid Ali

sajidali.qau@gmail.com

Munir Ahmed

irmunir@gmail.com

1 Department of Statistics, Islamabad Model College for Boys, Sector F-10/4, Islamabad, Pakistan

2 School of Business and Economics, Lahore, Pakistan

3 Department of Mathematics, Islamabad Model College for Boys, Sector F-10/4, Islamabad, Pakistan depends only on the mixture and not on the total amount of the mixture. The factor space is a $(q-1)$-dimensional regular simplex $S_{q-1}$ :

$S_{q-1}=\left\{x:\left(x_{1}, x_{2}, \ldots, x_{q}\right) \mid \sum_{i=1}^{q} x_{i}=1, x_{i} \geq 0\right\}$.

There are various mixture models available in the literature, and for the estimation of their parameters, many mixture designs have been proposed. Simplex-centroid design is the simplest and widely used mixture design by the practitioners. The main feature of this article is the construction of simplex-centroid mixture design with the real mixture blends, though with less efficiency. The design points in a simplex-centroid design do not completely explore the whole mixture region, whereas in our proposed designs, the design points fall uniformly inside the mixture space and explore it in true sense. Prescott (1998) shrunk the co-ordinates of design points towards the centroid after their re-parameterization, using orthogonally blocked mixture designs. The resulting designs were composed of full mixture blends, falling inside the simplex. The designs were less efficient as compared to the original design.

The article is organized as follows. First simplex-centroid design is reviewed. Next, the concept of shrinkage design and the re-parameterization of co-ordinate system for three and fours components are discussed. Then, the shrinkage simplex-centroid designs for three and four components are constructed and their $D$ - and $G$-efficiencies are compared. The proposed designs are further used to develop component-amount designs by their projection. Finally, the application of shrinkage design is given and conclusions are made. 

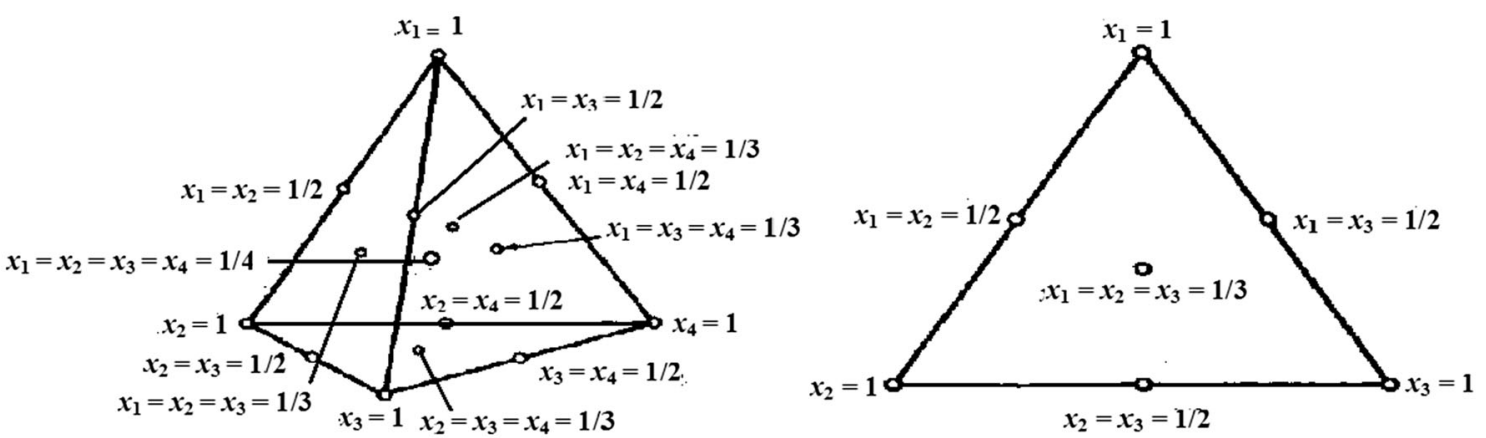

Fig. 1 Simplex-centroid designs for three and four components

Table 1 Simplex-centroid design for three components

\begin{tabular}{llll}
\hline Run & $x_{1}$ & $x_{2}$ & $x_{3}$ \\
\hline 1 & 1 & 0 & 0 \\
2 & 0 & 1 & 0 \\
3 & 0 & 0 & 1 \\
4 & $1 / 2$ & $1 / 2$ & 0 \\
5 & $1 / 2$ & 0 & $1 / 2$ \\
6 & 0 & $1 / 2$ & $1 / 2$ \\
7 & $1 / 3$ & $1 / 3$ & $1 / 3$ \\
\hline
\end{tabular}

\section{Review of simplex-centroid design}

Scheffé (1963) introduced the simplex-centroid design, where only such mixtures are considered in which the components presented have equal proportions. It has $2^{q}-1$ design points. These design points have ${\underset{1}{C}}_{1}^{q}$ permutations of $(1,0, \ldots, 0)$, the $\underset{2}{\stackrel{q}{C}}$ permutations of $\left(\frac{1}{2}, \frac{1}{2}, 0, \ldots, 0\right)$, the $\underset{3}{C}$ permutations of $\left(\frac{1}{3}, \frac{1}{3}, \frac{1}{3}, 0, \ldots, 0\right)$, and so on, and the overall centroid $\left(\frac{1}{q}, \frac{1}{q}, \frac{1}{q}, \ldots, \frac{1}{q}\right)$. Such mixtures are located at the centroid of the $(q-1)$-dimensional simplex and the centroids of all the lower dimensional simplexes within the $(q-1)$-dimensional simplex. Responses are collected at the design points and a polynomial is fitted. The design points in the simplex-centroid design will support the polynomial equation. The general form of the quadratic Scheffé polynomial equation is

$E(Y)=\sum_{i=1}^{q} \beta_{i} X_{i}+\sum_{i=1}^{q-1} \sum_{j=i+1}^{q} \beta_{i j} X_{i} X_{j}$

Simplex-centroid designs for three and four components are given in Tables 1 and 2, and are depicted by Fig. 1a and b.

In real-life situations, we always use a mixture that has at least a minimum proportion of all the ingredients present. Therefore, we need to construct the optimal designs
Table 2 Simplex-centroid design for four components

\begin{tabular}{lllll}
\hline Run & $x_{1}$ & $x_{2}$ & $x_{3}$ & $x_{4}$ \\
\hline 1 & 1 & 0 & 0 & 0 \\
2 & 0 & 1 & 0 & 0 \\
3 & 0 & 0 & 1 & 0 \\
4 & 0 & 0 & 0 & 1 \\
5 & $1 / 2$ & $1 / 2$ & 0 & 0 \\
6 & $1 / 2$ & 0 & $1 / 2$ & 0 \\
7 & $1 / 2$ & 0 & 0 & $1 / 2$ \\
8 & 0 & $1 / 2$ & $1 / 2$ & 0 \\
9 & 0 & $1 / 2$ & 0 & $1 / 2$ \\
10 & 0 & 0 & $1 / 2$ & $1 / 2$ \\
11 & $1 / 3$ & $1 / 3$ & $1 / 3$ & 0 \\
12 & $1 / 3$ & $1 / 3$ & 0 & $1 / 3$ \\
13 & $1 / 3$ & 0 & $1 / 3$ & $1 / 3$ \\
14 & 0 & $1 / 3$ & $1 / 3$ & $1 / 3$ \\
15 & $1 / 4$ & $1 / 4$ & $1 / 4$ & $1 / 4$ \\
\hline
\end{tabular}

with complete mixture blends. The standard simplex design is a boundary point design with the exception of the overall centroid that is all the other points are on the boundary of the simplex. We propose the construction of three and four components' simplex-centroid designs by the shrinkage of design points towards its centroid and call them shrinkage simplex-centroid designs.

\section{Shrinkage design}

The pioneering work on this issue was done by Prescott (1998). He constructed nearly optimal designs for Scheffé's quadratic mixture model with three and four components, using Latin square-based orthogonal blocking scheme. Such optimal designs although have complete mixture compositions, but are less efficient. Aggarwal et al. (2011) proposed nearly D-, A-, and E-optimal designs for Scheffé's, Kronecker, Becker's, and Darroch and Waller's quadratic mixture models in four components, using F-square-based orthogonal blocking scheme. Hasan and 


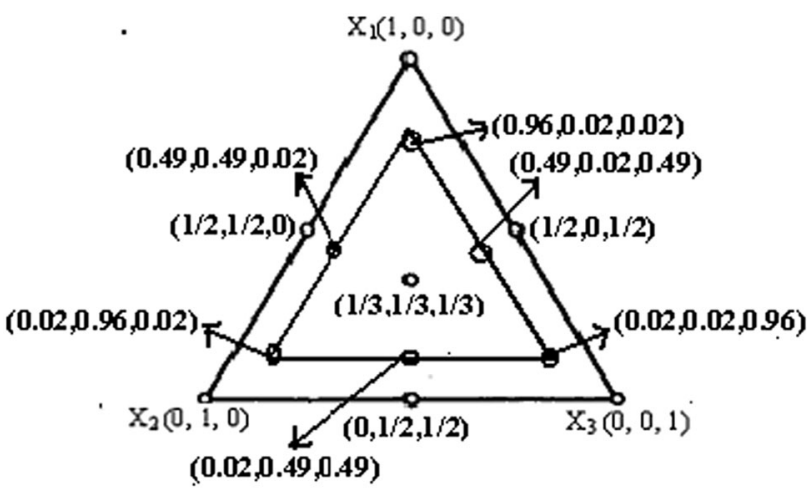

Fig. 2 Simplex-centroid design and shrinkage simplex-centroid design with $s=0.05$

Khan $(2011,2012)$ proposed nearly D-, A-, and E-optimal orthogonally blocked designs in three components using Scheffé's and Kronecker mixture models. All the above references deal with the construction of shrinkage designs in orthogonal blocks. We have adapted the same methodology for the construction of shrinkage optimal design using simplex-centroid design, where the mixture blends are not in orthogonal blocks.

\section{Re-parameterization of the co-ordinate system}

Consider a mixture region in $(q-1)$ dimensions and denote the co-ordinates in the region by the symbols $a, b, c, \ldots$, such that $a+b+c+\cdots=1$. The orthogonal blocks containing pairs of Latin squares provide mathematical expression for the information matrix $X^{\prime} X$ in terms of the symbols $a, b, c, \ldots$ Using any optimal criteria, we can determine the optimal values of $a, b, c, \ldots$ Prescott (1998) discussed the re-parameterization of co-ordinates of points in a mixture region and constructed nearly D-optimal designs. We review this re-parameterization method for the construction of Shrinkage simplex-centroid design in three and four components.

\section{(1) Three components' mixture}

Consider a two-dimensional simplex formed by three components, as given in Fig. 2. Take any design point $\mathrm{P}(a$, $b, c)$ in the simplex, such that $a \geq b \geq c$. Express the coordinates $a, b$, and $c$ in terms of $s$ and $f$, where $s$ is the shrinkage parameter with $f$ be a co-ordinate of the point on the edge of simplex, and $(1 / 3,1 / 3,1 / 3)$ is the centroid of the simplex:

$$
\begin{aligned}
& a=(1-s) f+\frac{s}{3} \\
& b=(1-s)(1-f)+\frac{s}{3} \\
& c=\frac{s}{3} .
\end{aligned}
$$

For instance, when $s=0$, the point $\mathrm{P}(a, b, c)$ reduces to $\mathrm{P}(f, 1-f, 0,0)$, falling on the edge of the simplex.

\section{(2) Four components' mixture}

We re-parameterize a point $P$ with the co-ordinates $(a, b, c, d)$ in a three-dimensional simplex, where $a \geq b \geq c \geq d$. As a special case when $c=d$, the co-ordinates $(a, b, c, d)$ of the point $P$, expressed as a function of $(f, s)$, are given as follows:

$$
\begin{aligned}
& a=(1-s) f+s / 4 \\
& b=(1-s)(1-f)+s / 4 \\
& c=d=s / 4 .
\end{aligned}
$$

The extension of re-parameterization to mixture experiments with more than four components is very simple.

\section{Shrinkage simplex-centroid designs for three and four components}

We construct shrinkage simplex-centroid design by considering quadratic Scheffé's mixture models in three and four components. The $D$-efficiency of shrinkage designs is obtained with regard to the D-criterion, where $D=\left|X^{\prime} X\right|^{1 / P}$ and $P$ denotes the number of parameters in the model:

D-efficiency $=\left|X^{\prime} X\right|^{1 / P} /\left|X^{\prime} X\right|_{o}^{1 / P} \times 100$.

Here, $\left|X^{\prime} X\right|_{o}^{1 / P}$ is the value of $D$ for non-shrinkage design. Furthermore, the prediction capability of a design can be assessed using G-optimality criterion, which searches for the design that minimizes the maximum prediction variance over the experimental region. A design criterion related to G-optimality is $G$-efficiency, defined as

G-efficiency $=100 \times\left(\frac{p / n}{\mathrm{MPV}}\right)$,

where $p / n$ is the average prediction variance (APV) and MPV is the maximum prediction variance. We evaluate $D$ and $G$-efficiencies of shrinkage designs for several values of parameter $s$, for a quadratic Scheffé's model. The three components' quadratic Scheffé's model is

$\begin{aligned} E(y)= & \beta_{1} x_{1}+\beta_{2} x_{2}+\beta_{3} x_{3}+\beta_{12} x_{1} x_{2}+\beta_{13} x_{1} x_{3} \\ & +\beta_{23} x_{2} x_{3} .\end{aligned}$

The value of $D$ for simplex-centroid design, given in Table 1, for three component quadratic Scheffé's model is 0.27049 . Now, consider that the shrinkage parameter $s=0.05$. Using re-parameterized co-ordinates given in Eq. (1), the shrinkage simplex-centroid design is obtained as follows. 
Table 3 Shrinkage simplexcentroid design with the parameter $s=0.05$

\begin{tabular}{llll}
\hline Run & $x_{1}$ & $x_{2}$ & $x_{3}$ \\
\hline 1 & 0.96 & 0.02 & 0.02 \\
2 & 0.02 & 0.96 & 0.02 \\
3 & 0.02 & 0.02 & 0.96 \\
4 & 0.49 & 0.49 & 0.02 \\
5 & 0.49 & 0.02 & 0.49 \\
6 & 0.02 & 0.49 & 0.49 \\
7 & $1 / 3$ & $1 / 3$ & $1 / 3$ \\
\hline
\end{tabular}

Table $4 D$-efficiency of shrinkage simplex-centroid designs for $q=3$

\begin{tabular}{lllc}
\hline$s$ & $\left|X^{\prime} X\right|^{1 / p}$ & $\left|X^{\prime} X\right|_{0}^{1 / p}$ & $D$-efficiency \\
\hline 0.00 & 0.270492 & 0.270492 & 100 \\
0.05 & 0.229272 & 0.270492 & 85 \\
0.10 & 0.201085 & 0.270492 & 74 \\
\hline
\end{tabular}

Table $5 G$-efficiency of shrinkage simplex-centroid designs for $q=3$

\begin{tabular}{llll}
\hline$s$ & APV & MPV & $G$-efficiency \\
\hline 0.00 & 0.857 & 0.992 & 86.4 \\
0.05 & 0.857 & 0.992 & 86.4 \\
0.10 & 0.857 & 0.992 & 86.4 \\
\hline
\end{tabular}

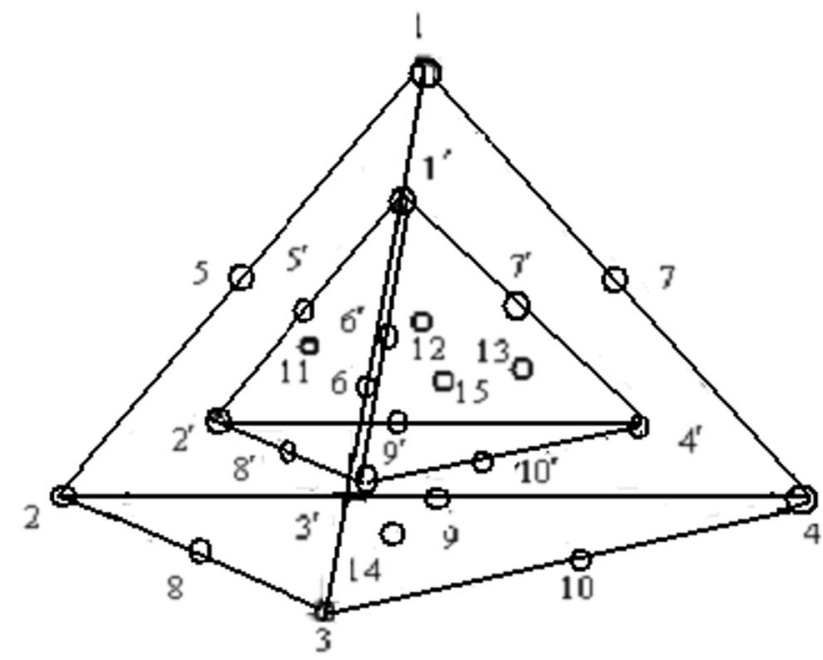

Fig. 3 Simplex-centroid design and shrinkage simplex-centroid design for $q=4$ with $s=0.05$ (simple number shows runs in simplex-centroid design and the numbers with primes show runs in shrinkage simplex-centroid design)

The value of $D$ for shrinkage simplex-centroid design given in Table 3 for quadratic Scheffé's model is 0.2293. Using $D$-efficiency criterion given in Eq. (3), the efficiency of the above design is $85 \%$. Comparing the design in Table 3
Table 6 Shrinkage simplex-centroid design for four components with $s=0.05$

\begin{tabular}{lllll}
\hline Run & $x_{1}$ & $x_{2}$ & $x_{3}$ & $x_{4}$ \\
\hline 1 & 0.9625 & 0.0125 & 0.0125 & 0.0125 \\
2 & 0.0125 & 0.9625 & 0.0125 & 0.0125 \\
3 & 0.0125 & 0.0125 & 0.9625 & 0.0125 \\
4 & 0.0125 & 0.0125 & 0.0125 & 0.9625 \\
5 & 0.4875 & 0.4875 & 0.0125 & 0.0125 \\
6 & 0.4875 & 0.0125 & 0.4875 & 0.0125 \\
7 & 0.4875 & 0.0125 & 0.0125 & 0.4875 \\
8 & 0.0125 & 0.4875 & 0.4875 & 0.0125 \\
9 & 0.0125 & 0.4875 & 0.0125 & 0.4875 \\
10 & 0.0125 & 0.0125 & 0.4875 & 0.4875 \\
11 & 0.32915 & 0.32915 & 0.3215 & 0.0125 \\
12 & 0.32915 & 0.32915 & 0.0125 & 0.32915 \\
13 & 0.32915 & 0.0125 & 0.32915 & 0.32915 \\
14 & 0.0125 & 0.32915 & 0.32915 & 0.32915 \\
15 & 0.2500 & 0.2500 & 0.2500 & 0.2500 \\
\hline
\end{tabular}

Table $7 D$-efficiency of shrinkage simplex-centroid designs for $q=4$

\begin{tabular}{llll}
\hline$s$ & $\left|X^{\prime} X\right|^{1 / p}$ & $\left|X^{\prime} X\right|_{0}^{1 / p}$ & $D$-efficiency \\
\hline 0.00 & 0.232169 & 0.232169 & 100 \\
0.05 & 0.179471 & 0.232169 & 77.3 \\
0.10 & 0.169251 & 0.232169 & 73.0 \\
\hline
\end{tabular}

Table $8 G$-efficiency of shrinkage simplex-centroid designs for $q=4$

\begin{tabular}{llll}
\hline$s$ & APV & MPV & $G$-efficiency \\
\hline 0.00 & 0.667 & 0.978 & 68.2 \\
0.05 & 0.667 & 0.977 & 68.2 \\
0.10 & 0.667 & 0.977 & 68.2 \\
\hline
\end{tabular}

with the simplex-centroid design in Table 1, it is clear that the constructed design is a true mixture design with full mixture blends, though less efficient. Table 4 gives $D$-efficiency of shrinkage simplex-centroid designs for several values of $s$. In Table 5, we have $G$-efficiency of the designs for different values of shrinkage parameter $s$ (Fig. 3).

Next, we consider four components' quadratic Scheffé mixture model:

$$
\begin{aligned}
E(y)= & \beta_{1} x_{1}+\beta_{2} x_{2}+\beta_{3} x_{3}+\beta_{4} x_{4}+\beta_{12} x_{1} x_{2}+\beta_{13} x_{1} x_{3} \\
& +\beta_{14} x_{1} x_{4}+\beta_{23} x_{2} x_{3}+\beta_{24} x_{2} x_{4}+\beta_{34} x_{3} x_{4} .
\end{aligned}
$$

The value of $D$ for simplex-centroid design, given in Table 2, for four components' quadratic Scheffé's model is 0.2322. Now, shrink design in Table 2 is towards the 
centroid, with the shrinkage parameter $s=0.05$ and reparameterizes the co-ordinates using Eq. (2). The resulting shrinkage simplex-centroid design is given as follows.

The value of $D$ for shrinkage simplex-centroid design, given in Table 6, for quadratic Scheffé's model is 0.1795. Using $D$-efficiency criterion given in Eq. (3), the efficiency of the above design is $77.3 \%$. Tables 7 and 8 provide $D$ and $G$-efficiencies, respectively, for shrinkage simplexcentroid designs with several values of $s$.

Hence, comparing the design in Table 6 with the simplex-centroid design in Table 2, the constructed design is a true mixture design, containing full mixture blends with some compromise on its efficiency.

\section{Construction of component-amount design via projection of shrinkage simplex-centroid design}

A mixture-amount experiment is a type of mixture experiment that is performed at two or more levels of total amount. The response is assumed to be dependent upon the individual proportions of components in the blend and also on its amount. The effect on the response after varying mixture component proportions and the total amount of the mixture is measured by fitting a mixture-amount model to the design. The design for fitting mixture-amount model is called mixture-amount design, developed by Piepel and Cornell (1985). Piepel (1988) modified that model to accommodate zero-amount condition. The alternative model uses the actual amounts of the ingredients denoted by $a_{1}, a_{2}, \ldots, a_{q}$, such that $a_{1}+a_{2}+\cdots+a_{q}=A$. The proportions $x_{i}$ are related to the amount $a_{i}$ through $x_{i}=a_{i} / A$. This is called component-amount model:

$E(y)=\alpha_{0}+\sum_{i=1}^{q} \alpha_{i} a_{i}+\sum_{i=1}^{q}\left(\alpha_{i i} a_{i}^{2}+\sum_{i\langle j}^{q} \alpha_{i j} a_{i} a_{j}\right)$.

Prescott and Draper $(2004,2008)$ discussed the construction of designs for component-amount models by projecting standard symmetric mixture designs (simplexlattice and simplex-centroid designs) in lower dimensions. The method involved the collapsing of the standard symmetric mixture designs by the removal of their one or more columns. It leads to a set of symmetric designs for each of the several levels of the amount $A$. We construct component-amount designs by the projection of shrinkage simplex-centroid designs, as given in "Shrinkage design".

The component-amount design formed by Prescott and Draper $(2004,2008)$, through projection of simplex-centroid design, given in Table 2, is provided in Table 9.

The above component-amount design has the value $D=0.21825$ and its $G$-efficiency is $68.2 \%$. The design is
Table 9 Simplex-centroid design for four components

\begin{tabular}{lllll}
\hline Run & $a_{1}$ & $a_{2}$ & $a_{3}$ & $A$ \\
\hline 1 & 1 & 0 & 0 & 1 \\
2 & 0 & 1 & 0 & 1 \\
3 & 0 & 0 & 1 & 1 \\
4 & 0 & 0 & 0 & 0 \\
5 & $1 / 2$ & $1 / 2$ & 0 & 1 \\
6 & $1 / 2$ & 0 & $1 / 2$ & 1 \\
7 & $1 / 2$ & 0 & 0 & $1 / 2$ \\
8 & 0 & $1 / 2$ & $1 / 2$ & 1 \\
9 & 0 & $1 / 2$ & 0 & $1 / 2$ \\
10 & 0 & 0 & $1 / 2$ & $1 / 2$ \\
11 & $1 / 3$ & $1 / 3$ & $1 / 3$ & 1 \\
12 & $1 / 3$ & $1 / 3$ & 0 & $2 / 3$ \\
13 & $1 / 3$ & 0 & $1 / 3$ & $2 / 3$ \\
14 & 0 & $1 / 3$ & $1 / 3$ & $2 / 3$ \\
15 & $1 / 4$ & $1 / 4$ & $1 / 4$ & $3 / 4$ \\
\hline
\end{tabular}

Table 10 Shrinkage simplex-centroid component-amount design for three components

\begin{tabular}{lllll}
\hline Run & $a_{1}$ & $a_{2}$ & $a_{3}$ & $A$ \\
\hline 1 & 0.9625 & 0.0125 & 0.0125 & 0.9875 \\
2 & 0.0125 & 0.9625 & 0.0125 & 0.9875 \\
3 & 0.0125 & 0.0125 & 0.9625 & 0.9875 \\
4 & 0.0125 & 0.0125 & 0.0125 & 0.0375 \\
5 & 0.4875 & 0.4875 & 0.0125 & 0.9875 \\
6 & 0.4875 & 0.0125 & 0.4875 & 0.9875 \\
7 & 0.4875 & 0.0125 & 0.0125 & 0.5125 \\
8 & 0.0125 & 0.4875 & 0.4875 & 0.9875 \\
9 & 0.0125 & 0.4875 & 0.0125 & 0.5125 \\
10 & 0.0125 & 0.0125 & 0.4875 & 0.5125 \\
11 & 0.32915 & 0.32915 & 0.32915 & 0.9875 \\
12 & 0.32915 & 0.32915 & 0.0125 & 0.6708 \\
13 & 0.32915 & 0.0125 & 0.32915 & 0.6708 \\
14 & 0.01250 & 0.32915 & 0.32915 & 0.6708 \\
15 & 0.2500 & 0.2500 & 0.2500 & 0.7500 \\
\hline
\end{tabular}

composed of incomplete mixture blends with different levels of amounts except the centroid point as a complete mixture blend. For real-life situations, we need componentamount designs which have full mixture blends with at least a minimum amount of each component in the mixture. Such designs can be obtained by the projection of shrinkage simplex-centroid designs.

We project the design, given in Table 6 , to lower dimension by deleting any column. Let the column for $x_{4}$ in Table 6 is deleted. The resulting component-amount design in three components has the levels of amount $A=0.0375$, $0.5125,0.6708,0.9875$, and 0.7500 with replicates $1,3,3$, 7 , and 1 and is given in Table 10. This shrinkage 


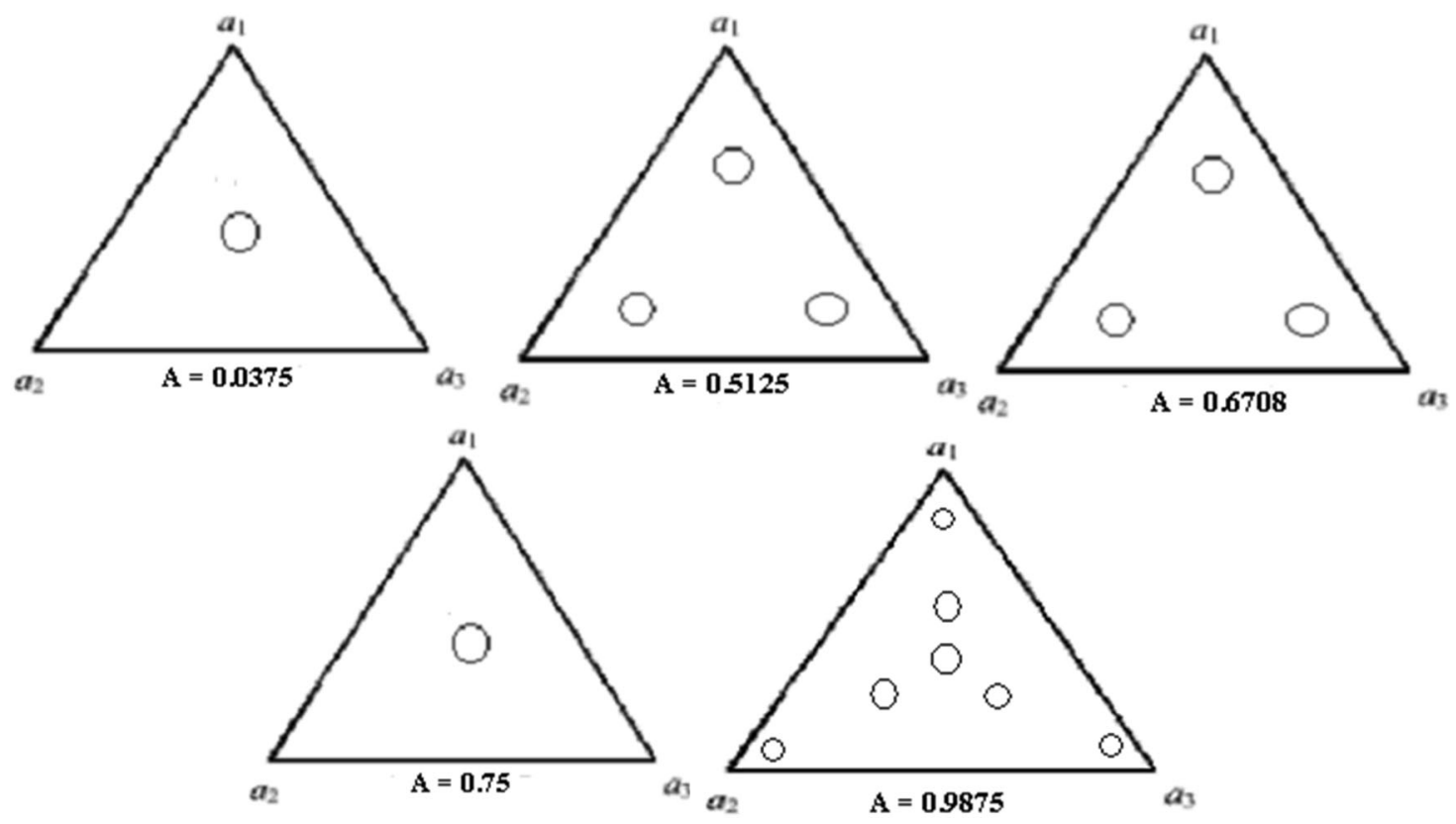

Fig. 4 Structure for the component-amount design in $a_{1}, a_{2}$, and $a_{3}$ simplex, formed by the projection of shrinkage simplex-centroid design with the shrinkage parameter $s=0.05$

component-amount design has the $D$-efficiency $91.2 \%$ and the $G$-efficiency design $68.2 \%$. On comparison of this design with the design in Table 9, it can be noted that the constructed design is composed of full mixture blends, although it has less efficiency (Fig. 4).

\section{Example: diazepam solubility experiment}

We take an example, given in Smith (Smith 2005, p. 54). Belloto et al. (1985) were interested in studying the solubility of drug diazepam in mixtures of ethanol, propylene glycol, and water. It was desired to develop a mixture model that would predict the diazepam in any mixture of solvents in a three-dimensional simplex. We first choose simplex-centroid design given in Table 1 and augment it with two replicates at each of the pure blends and with three axial check blends.

The $G$-efficiency of the design is $63.14 \%$. We shrink augmented simplex-centroid design, given in Table 11, using the shrinkage parameter $s=0.1$. The $G$-efficiency of the design is again $63.14 \%$, while its $D$-efficiency is $74.4 \%$. Therefore, mixture design with full mixture blends can be used to develop a mixture model with the stable $G$-efficiency that would predict the diazepam in any mixture of solvents in a three-dimensional simplex (Fig. 5).
Table 11 Augmented simplex-centroid design for three components

\begin{tabular}{lllll}
\hline Run & $x_{1}$ & $x_{2}$ & $x_{3}$ & $y$ \\
& Ethanol & Glycol & Water & Solubility $(\mathrm{mg} / \mathrm{ml})$ \\
\hline 1 & 1 & 0 & 0 & 27.8 \\
2 & 0 & 1 & 0 & 7.42 \\
3 & 0 & 0 & 1 & 0.048 \\
4 & $1 / 2$ & $1 / 2$ & 0 & 27.0 \\
5 & $1 / 2$ & 0 & $1 / 2$ & 6.02 \\
6 & 0 & $1 / 2$ & $1 / 2$ & 0.61 \\
7 & $4 / 6$ & $1 / 6$ & $1 / 6$ & 28.0 \\
8 & $1 / 6$ & $4 / 6$ & $1 / 6$ & 13.0 \\
9 & $1 / 6$ & $1 / 6$ & $4 / 6$ & 0.408 \\
10 & $1 / 3$ & $1 / 3$ & $1 / 3$ & 9.52 \\
11 & 0 & 1 & 0 & 7.42 \\
12 & 1 & 0 & 0 & 27.8 \\
13 & 0 & 0 & 1 & 0.048 \\
\hline
\end{tabular}

\section{Conclusion}

Simplex-centroid design is a widely used mixture design for quadratic mixture model. This classical design does not contain full mixture blends except centroid. In real-life situation, a mixture always has a least proportion of every component. In the current study, shrinkage designs are 

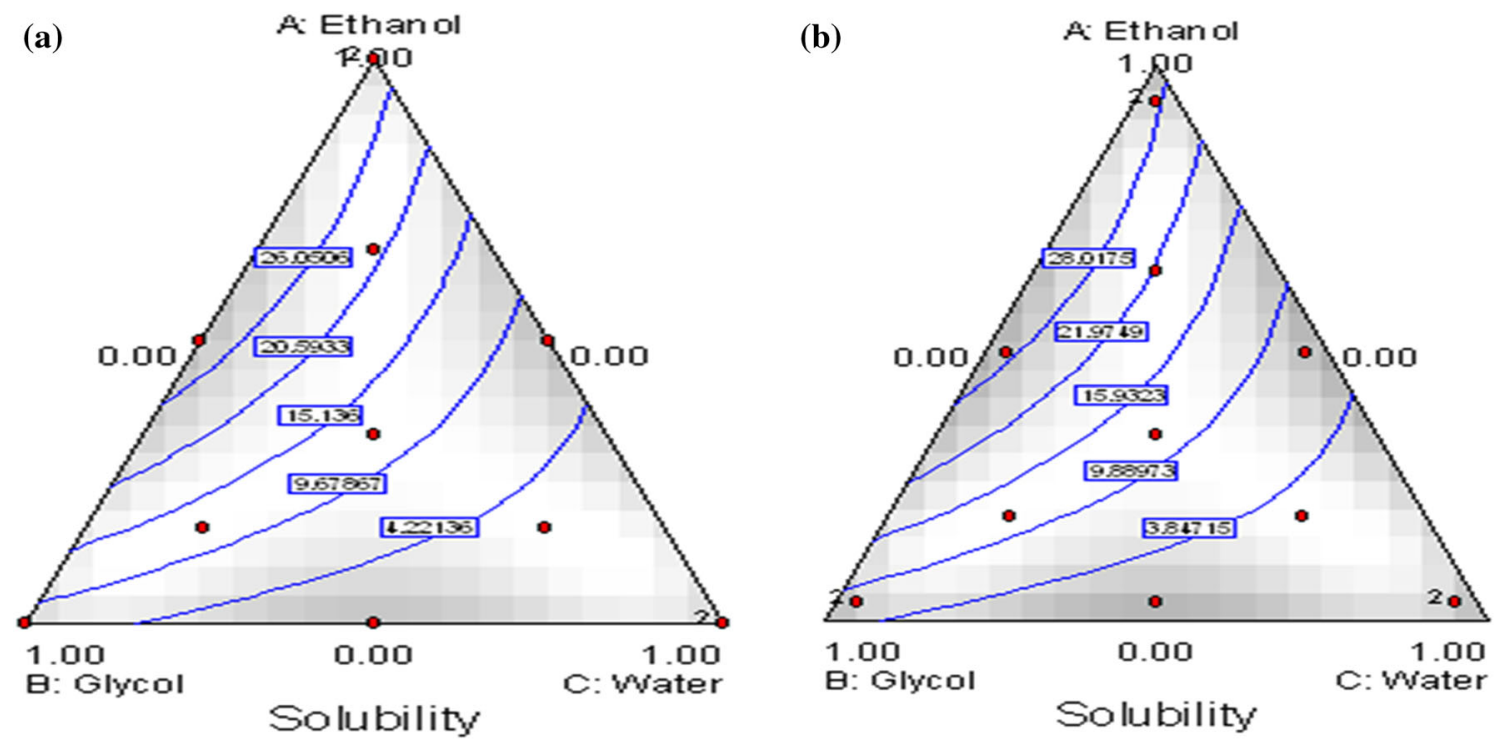

Fig. 5 a Contour plot of predicted solubility using the design in Table 11 and $\mathbf{b}$ contour plot of predicted solubility using shrinkage design with parameter $s=0.1$

constructed from simplex-centroid designs. The proposed shrinkage simplex-centroid designs are the real-life mixture designs, although they have less efficiency as compared to the simplex-centroid designs available in the literature. This stability in $G$-efficiency of these designs shows that the prediction capability of the model does not change by shrinking conventional simplex-centroid design towards the centroid. Therefore, compromising on the loss in D-optimality of simplex-centroid design, mixture designs with full mixture blends and with the stable G-optimality can be constructed.

Open Access This article is distributed under the terms of the Creative Commons Attribution 4.0 International License (http://crea tivecommons.org/licenses/by/4.0/), which permits unrestricted use, distribution, and reproduction in any medium, provided you give appropriate credit to the original author(s) and the source, provide a link to the Creative Commons license, and indicate if changes were made.

\section{References}

Aggarwal ML, Singh P, Sarin V, Husain B (2011) Nearly optimal orthogonally blocked designs for four mixture components in three and four components based on F-squares. Commun Stat Simul 40(1):165-183
Belloto RJ, Dean AM, Moustafa MA, Molokhia AM, Gouda MW, Sokoloski TD (1985) Statistical techniques applied to solubility predictions and pharmaceutical formulations: an approach to problem solving using mixture response surface methodology. Int J Pharm 23:195-207

Hasan T, Khan MF (2011) Nearly A- and E-optimal orthogonally blocked designs for Scheffé's quadratic mixture model with three components. Proc Pak Acad Sci 48(2):101-106

Hasan T, Khan MF (2012) Nearly optimal orthogonally blocked designs for second degree K-model in three components. Pak J Stat 28(2): 177-187

Piepel GF (1988) A note on models for mixture-amount experiments when the total amount takes a zero value. Technometrics 30:449-450

Piepel GF, Cornell JA (1985) Models for mixture experiments when the response depends on the total amount. Technometrics 27:219-227

Prescott P (1998) Nearly optimal orthogonally blocked designs for a quadratic mixture model with $q$ components. Commun Stat Theory Methods 27(10):2259-2580

Prescott P, Draper NR (2004) Mixture component-amount designs via projections, including orthogonally blocked designs. J Qual Technol 36:413-431

Prescott P, Draper NR (2008) D-optimal component-amount designs for quadratic and cubic models. J Appl Stat 35(7):739-749

Scheffé H (1963) Simplex-centroid designs for experiments with mixtures. J R Stat Soc B 25:235-263

Smith WF (2005) Experimental design for formulation. ASA-SIAM Series on statistics and applied probability. SIAM/American Statistical Association, Philadelphia/Alexandria 